\title{
Role of balloon dilatation of the aortic valve in pregnant patients with severe aortic stenosis
}

\author{
A P Banning, J F Pearson, R J C Hall
}

\begin{abstract}
The outcome of unrelieved severe symptomatic aortic stenosis in pregnancy is poor. Though the valve lesion can be corrected surgically before delivery at a low risk to the mother, cardiopulmonary bypass during pregnancy carries a high risk to the fetus. Two patients in the second trimester of pregnancy were successfully managed with balloon dilatation of the aortic valve. Both delivered healthy infants and were well a year later. Balloon dilatation of the aortic valve is a useful palliative procedure in the management of pregnant women with severe aortic stenosis.
\end{abstract}

(Br Heart f 1993;70:544-545)

Severe aortic stenosis in patients who become pregnant is an unusual and demanding clinical problem. Altered haemodynamic function in pregnancy combined with the relatively fixed cardiac output caused by the stenosed valve leads to a significant risk of maternal death. ${ }^{1}$ Cardiopulmonary bypass carries a small maternal risk but a very high fetal risk. ${ }^{2}$ Balloon dilatation of the aortic valve performed during pregnancy has been reported twice before ${ }^{34}$ and we describe two further cases in which it was successful.

\section{Case reports}

CASE 1

A 26-year-old primigravida presented at 14 weeks' gestation having been referred from her antenatal booking clinic. She had been investigated at the age of 9 and diagnosed as having a bicuspid aortic valve with mild aortic stenosis, but she was subsequently lost to follow up until she became pregnant. During early pregnancy she had complained of mild exertional dyspnoea and had also experienced two episodes of near syncope.

Examination showed a pulse of 90 beats per minute and blood pressure of $90 / 75 \mathrm{~mm}$ Hg. An ejection click was followed by a harsh ejection systolic murmur, audible in the left third intercostal space and there was a sustained left ventricular impulse. Cross sectional echocardiography showed a grossly hypertrophied left ventricle and a thickened mobile aortic valve. The aortic valve was not significantly calcified and the Dopplerderived peak gradient across it was 120-140 mm Hg.
After discussion with the cardiothoracic surgeons, radiologists, and obstetricians, percutaneous balloon dilatation of the aortic valve was planned with cardiothoracic surgical standby. Catherisation was performed via the right femoral artery and right femoral vein. To minimise $x$ ray exposure to the uterus, lead shields were placed around the abdomen and no angiograms were taken. A peak to peak gradient of $128 \mathrm{~mm} \mathrm{Hg}$ was measured across the aortic valve and the valve was subsequently dilated with two inflations of a $20 \mathrm{~mm}$ diameter Triad balloon (Mansfield Scientific). After the second inflation the systemic blood pressure rose from 90/74 to $112 / 52 \mathrm{~mm} \mathrm{Hg}$ and the peak to peak aortic gradient fell to $50 \mathrm{~mm} \mathrm{Hg}$. An $x$ ray film badge on her abdomen recorded a total radiation dose of $1.68 \mathrm{mSv}$ and a thermoluminescent chip placed on the patient's lumbar region recorded a dose of only $1.04 \mathrm{mSv}$.

Doppler echocardiography after the procedure showed moderate aortic regurgitation and a gradient across the aortic valve of 60 $\mathrm{mm} \mathrm{Hg}$. She was discharged two days later and remained symptom free throughout the rest of her pregnancy. She was admitted at $\mathbf{4 1}$ weeks' gestation and subsequently a healthy infant was delivered under general anaesthesia by elective caesarean section.

At routine follow up one year after the procedure she was symptom free and the findings on Doppler echocardiography were unchanged.

CASE 2

A 19-year-old primigravida presented at 16 weeks' gestation complaining of worsening exertional dyspnoea and light-headed episodes on exercise. A congenitally bicuspid aortic valve had been diagnosed 11 years previously and she had become pregnant while awaiting elective aortic valve replacement, after the development of mild exertional dyspnoea and a peak gradient of $90 \mathrm{~mm} \mathrm{Hg}$ across the aortic valve, measured by Doppler echocardiography.

Cardiac catheterisation was undertaken through the right femoral artery and brachial vein. A peak to peak gradient of $123 \mathrm{~mm} \mathrm{Hg}$ was demonstrated between left ventricle and aorta. The aortic valve was dilated with a 15 mm diameter balloon initially and subsequently with a $20 \mathrm{~mm}$ diameter balloon. Each balloon was inflated once and after dilatation the peak to peak gradient fell to $60 \mathrm{~mm} \mathrm{Hg}$.

Her symptoms improved and at 36 weeks' gestation a healthy male infant was delivered \\ Departments of \\ Obstetrics, University \\ Cardiff \\ R J C Hall \\ Correspondence to Dr A P \\ Banning, Department of \\ Cardiology, University of \\ 4 June 1993.
}


by elective caesarean section. The Dopplerestimated peak gradient remained approximately $60 \mathrm{~mm} \mathrm{Hg}$ postpartum and this was unchanged at follow up one year later.

\section{Discussion}

The physiological changes induced by pregnancy include increased cardiac output and hypervolaemia. This change in cardiac output is, however, limited in patients with severe aortic stenosis by the presence of the stenosed valve and consequently the cardiac output is relatively fixed. Increases in cardiac output in response to exercise are limited and any significant fall in venous return can lead to a dramatic fall in cardiac output, syncope, and even sudden death. ${ }^{5}$

Pregnancy in patients with significant aortic stenosis has been reported infrequently and there is no single series large enough for a consensus to have been reached about what constitutes the ideal treatment. Traditionally the management of patients has been medical, with periods of prolonged hospitalisation for enforced bed rest. In 1978 Arias and Pineda ${ }^{1}$ reviewed reports of 38 pregnancies in 23 patients with aortic stenosis. They reported maternal mortality of $17 \cdot 4 \%(4 / 23)$ and fetal mortality of $34 \%$ (13/48). Termination of pregnancy offered little advantage: two maternal deaths occurred during five attempted therapeutic abortions (mortality 40\%), though these abortions were probably undertaken in those patients in whom continuation of the pregnancy was judged to be likely to carry the highest maternal risk.

Improvements in cardiac surgery and anaesthetics have considerably reduced the maternal risk of surgery. The estimated mortality is $1.5 \%,{ }^{2}$ which is only marginally higher than the risk of cardiopulmonary bypass in a non-pregnant patient. Both valve replacement and surgical valvotomy require cardiopulmonary bypass, which carries a high fetal risk $(9.5 \%){ }^{2}$ In patients in the second trimester of pregnancy these risks to the fetus are unacceptable and alternative management techniques have therefore been explored.

The use of percutaneous techniques in pregnant patients invokes the risks associated with the use of radiation. By using short screening times, lead shielding, and avoiding angiograms the uterine exposure to radiation can be kept to a minimum level. The exposure in our patient of $1.68 \mathrm{mSv}$ is less than the median dose absorbed by a patient after a single abdominal radiograph ${ }^{6}$ and also below the $2 \mathrm{mSv}$ effective dose limit for an occupationally exposed pregnant individual during nine months of pregnancy. ${ }^{7}$

The initial enthusiasm for balloon dilatation in older adults with calcific aortic stenosis has now been tempered by follow up studies showing that the duration of benefit to patients is only about six months and valve replacement is therefore generally recommended. ${ }^{8}$ Balloon dilatation of the aortic valve was successful in children with aortic stenosis when it was used to provide palliation while they grew, ${ }^{9}$ thus minimising the number of surgical procedures. The stenosed aortic valves of young adults are similar to those of children and dilatation is often successful. Successful balloon dilatation of the aortic valve during pregnancy has been reported twice before. ${ }^{34}$

Balloon dilatation of the aortic valve in pregnant patients is a safe and effective way of achieving a successful outcome to pregnancy. If clinically indicated, valve replacement can be undertaken electively postpartum.

We thank Dr J C Forfar for his assistance.

APB is a British Heart Foundation junior research fellow.

1 Arias F, Pineda J. Aortic stenosis and pregnancy. $\mathcal{F}$ Reprod Med 1978;20:229-32.

2 Bernal JM, Miralles PJ. Cardiac surgery with cardiopulmonary bypass during pregnancy. Obstet Gynecol Surv 1986;41:1-6.

3 Angel JL, Chapman C, Knuppel RA, et al. Percutaneous balloon valvoplasty in pregnancy. Obstet Gynecol 1988; 3:438-40.

4 McIvor RA. Percutaneous balloon aortic valvoplasty during pregnancy. Int $\mathcal{f}$ Cardiol 1991;32:1-4.

5 Clark S. Structural cardiac disease in pregnancy. In: Clark SL, Phelan JP, Cotton DB, eds. Critical care obstetrics. Oradell, New Jersey: Medical Ethics Company 1987; 92-113.

6 Dosimetry Working Party of the Institute of Physical Sciences in Medicine. National Protocol for patient dose measurement in diagnostic radiology. Oxford: National Radiation Protection Board, 1992:14.

7 ICRP. Recommendations of the International Commission on Radiological Protection. Annals of the ICRP 1991.

8 Hall RJC, Kirk R. Balloon dilatation of heart valves. $\mathrm{Br}$ Med $\mathcal{f}$ 1992;305:487-8.

9 O'Conner BK, Beekman RH, Rocchini AP, Rosenthal A. Intermediate term effectiveness of balloon valvoplasty of congenital aortic stenosis. Circulation 1991;84:732-8. 